\title{
Effects of Brain Parcellation on the Characterization of Topological Deterioration in Alzheimer's Disease
}

\author{
Zhanxiong $\mathrm{Wu}^{1,2}$, Dong $\mathrm{Xu}^{1,3}$, Thomas Potter ${ }^{2}$, Yingchun Zhang $^{2 *}$ and \\ the Alzheimer's Disease Neuroimaging Initiative ${ }^{\dagger}$ \\ ${ }^{1}$ School of Electronic Information, Hangzhou Dianzi University, Hangzhou, China, ${ }^{2}$ Department of Biomedical Engineering, \\ University of Houston, Houston, TX, United States, ${ }^{3}$ Zhejiang Key Laboratory of Equipment Electronics, Hangzhou, China
}

\section{OPEN ACCESS}

Edited by:

Beatrice Arosio,

University of Milan, Italy

Reviewed by:

Enrico Premi,

University of Brescia, Italy

Maria Marcella Lagana

Fondazione Don Carlo Gnocchi Onlus

(IRCCS), Italy

*Correspondence:

Yingchun Zhang

yzhang94@uh.edu

${ }^{\dagger}$ Data used in preparation of this article were obtained from the Alzheimer's Disease Neuroimaging

Initiative (ADNI) database

(adni.loni.usc.edu). As such, the

investigators within the ADNI

contributed to the design and

implementation of ADNI and/or provided data but did not participate in analysis or writing of this report. A complete listing of $A D N I$ investigators can be found at: http://adni./oni.usc.

edu/wp-content/uploads/

how_to_apply/

ADNI_Acknowledgement_List.pdf

Received: 13 December 2018 Accepted: 30 April 2019

Published: 21 May 2019

Citation:

Wu Z, Xu D, Potter T, Zhang Y and the

Alzheimer's Disease Neuroimaging

Initiative (2019) Effects of Brain

Parcellation on the Characterization of

Topological Deterioration in

Alzheimer's Disease.

Front. Aging Neurosci. 11:113.

doi: 10.3389/fnagi.2019.00113
Alzheimer's disease (AD) causes the progressive deterioration of neural connections, disrupting structural connectivity (SC) networks within the brain. Graph-based analyses of SC networks have shown that topological properties can reveal the course of $A D$ propagation. Different whole-brain parcellation schemes have been developed to define the nodes of these SC networks, although it remains unclear which scheme can best describe the AD-related deterioration of SC networks. In this study, four whole-brain parcellation schemes with different numbers of parcels were used to define SC network nodes. SC networks were constructed based on high angular resolution diffusion imaging (HARDI) tractography for a mixed cohort that includes 20 normal controls (NC), 20 early mild cognitive impairment (EMCl), 20 late mild cognitive impairment (LMCl), and 20 AD patients, from the Alzheimer's Disease Neuroimaging Initiative. Parcellation schemes investigated in this study include the OASIS-TRT-20 (62 regions), AAL (116 regions), HCP-MMP (180 regions), and Gordon-rsfMRI (333 regions), which have all been widely used for the construction of brain structural or functional connectivity networks. Topological characteristics of the SC networks, including the network strength, global efficiency, clustering coefficient, rich-club, characteristic path length, k-core, rich-club coefficient, and modularity, were fully investigated at the network level. Statistical analyses were performed on these metrics using Kruskal-Wallis tests to examine the group differences that were apparent at different stages of $A D$ progression. Results suggest that the HCP-MMP scheme is the most robust and sensitive to AD progression, while the OASIS-TRT-20 scheme is sensitive to group differences in network strength, global efficiency, k-core, and rich-club coefficient at $k$-levels from 18 and 39 . With the exception of the rich-club and modularity coefficients, AAL could not significantly identify group differences on other topological metrics. Further, the Gordon-rsfMRI atlas only significantly differentiates the groups on network strength, characteristic path length, k-core, and rich-club coefficient. Results show that the topological examination of SC networks with different parcellation schemes can provide important complementary AD-related information and thus contribute to a more accurate and earlier diagnosis of $A D$.

Keywords: Alzheimer's disease, mild cognitive impairment, high angular resolution diffusion imaging, structural connectivity network, fiber tracking 


\section{INTRODUCTION}

As the leading cause of dementia in elderly adults, Alzheimer's disease $(\mathrm{AD})$ is a progressive neurodegenerative disorder characterized by increasing cognitive and behavioral deficits (Mueller et al., 2005). Preceding AD, the mild cognitive impairment (MCI) phase presents with significant cognitive or behavioral deficits and an increased risk of developing dementia (Winblad et al., 2004; Jessen et al., 2014; Daianu et al., 2015; Mckenna et al., 2016). Understanding the physiological deterioration caused by MCI and AD provides an opportunity to develop future treatments and predict $\mathrm{AD}$ onset. Many postmortem histological and in-vivo imaging studies have demonstrated widespread white matter (WM) alterations in MCI and AD patients (Brun and Englund, 1986; Rose et al., 2000; Bozzali et al., 2002; Nir et al., 2015). The WM degeneration and neuronal death linked to $\mathrm{AD}$ progression then creates abnormal connectivity patterns between anatomically related brain regions (Lo et al., 2010). Specifically, demyelination and axonal degeneration cause drastic reductions in WM volume, which may contribute to alterations in structural connectivity (SC) network efficacy. Therefore, AD-related cognitive and behavioral deficits may be directly linked the disconnection of brain regions (Delbeuck et al., 2003; Sorg et al., 2009; Lo et al., 2010), such that altered SC topological patterns reflect the propagation stage of $\mathrm{AD}$.

High angular resolution diffusion imaging (HARDI) has provided an ability to extensively study brain networks in clinical neuroscience (Nguyen et al., 2018). The recent development of accurate and sophisticated HARDI-based tractography methods has encouraged the exploration of regional connectivity and topological network measures, which can quantify MCI and AD-linked brain changes. Graph theory has been frequently employed to detect SC network differences across normal control (NC), MCI, and AD groups, and a variety of topological measures sensitive to SC network disruption can be computed to reveal how $\mathrm{AD}$ affects the human connectome. Particular measures of interest include $k$-core, rich-club efficiency, nodal degree, characteristic path length, clustering coefficient, and global efficiency. To perform statistical analysis on SC networks, Kim et al. presented a multi-resolution analysis framework (Kim et al., 2015), in which a Wavelet representation of each anatomical connection was derived at multiple resolutions to analyze AD-related alterations. In Daianu et al. (2015), richclub properties at a range of degree thresholds were calculated, and their findings indicated that brain network disruptions occurred predominately in the low-degree $(<16)$ regions of the connectome in AD. In Daianu et al. (2013b), $k$-core was computed to understand the brain network breakdown caused by AD. In Lo et al. (2010), the alterations of various network properties were examined, indicating that $\mathrm{AD}$ patients exhibit shorter path lengths, decreased global efficiency, and reduced nodal efficiency. Yao et al. (2010) explored the characteristics of SC networks in MCI and $\mathrm{AD}$, finding that the MCI groups showed a loss of hub regions in the temporal lobe and altered interregional correlations, and that the topological measures of the MCI SC networks exhibited intermediate values. Most of these findings suggest that $\mathrm{AD}$ is related to the disruption of structural connectivity, which is characterized by the loss of richclub organization and network efficiency. Together, the findings suggest that $\mathrm{AD}$ is associated with a disrupted topological organization of SC networks, thus providing structural evidence for abnormalities in the SC network integrity of $\mathrm{AD}$ patients.

Graph-based analysis of brain structural networks provides a chance to understand how AD-linked structural connectivity abnormalities underlie the cognitive and behavioral deficits of patients. Specifically, the definition of network nodes is one of the most critical steps in network topological analysis, as it assigns the network structure and density for subsequent assessment. Different whole-brain parcellation schemes have been developed to define network nodes, although the effect that these schemes have on the detection of $\mathrm{AD}$ propagation stages remains unknown. Accurate brain parcellation provides a foundation for understanding the functional and structural organization of the human brain. During graph-based analysis of the SC networks derived from HARDI, brain parcellation is a key step for the construction of brain anatomical brain network architecture. This step is not trivial, however; the division of the cortex into different numbers of regions affects the structure of the SC network, such that the resulting topological properties of the generated SC network can be significantly changed by the scale of the chosen parcellation atlas (Proix et al., 2016). Considering that brain parcellation schemes are fundamental to the isolation and selection of brain regions, their application plays an important role in revealing the abnormal topological organization of SC networks in MCI and AD.

Cognitive studies have demonstrated that the cerebral cortex is comprised of distinct cortical areas that are interconnected through WM fibers (Sporns et al., 2004; delEtoile and Adeli, 2017). Network analysis represents cortical regions and their connections as a series of nodes and edges, respectively (Lo et al., 2010). Previous investigation have typically relied on a single type of whole-brain parcellation scheme to construct SC networks, such as the 96-region Harvard-Oxford atlas used in Shao et al. (2012), 113-region Harvard-Oxford atlas used in Zhan et al. (2015a), 162-region IIT3 atlas used in Kim et al. (2015), and 68region Desikan-Killiany atlas used in Daianu et al. (2013b) and Daianu et al. (2015). Each of these schemes presents a different number of parcels, and the effect this has on AD-related SC topological changes has not comprehensively characterized. In this study, SC networks are constructed for NC, early mild cognitive impairment (EMCI), late mild cognitive impairment (LMCI), and AD subjects based on HARDI tractography to fully characterize the manner in which patterns of SC network topological metrics change based on parcellation schemes. Four different whole-brain parcellation schemes over a range of parcellation scales $(62,116,180$, and 333 regions) were used to define SC network nodes: the OASIS-TRT-20 (62 regions) (Klein and Tourville, 2012), AAL (116 regions) (Tzourio-Mazoyer et al., 2002), HCP-MMP (180 regions) (Glasser et al., 2016), and Gordon-rsfMRI (333 regions) (Gordon et al., 2014). Edges were then estimated through deterministic fiber tracking based on orientation distribution function (ODF) fields, which was derived from HARDI images (Iturria-Medina et al., 2008; Descoteaux 
TABLE 1 | Demographics information for ANDI participants, arranged into NC, $\mathrm{EMCl}, \mathrm{LMCl}$, and AD groups.

\begin{tabular}{lllll}
\hline & NC & EMCI & LMCI & AD \\
\hline $\mathrm{N}$ & 20 & 20 & 20 & 20 \\
Gender & $8 \mathrm{M} / 12 \mathrm{~F}$ & $13 \mathrm{M} / 7 \mathrm{~F}$ & $11 \mathrm{M} / 9 \mathrm{~F}$ & $10 \mathrm{M} / 10 \mathrm{~F}$ \\
Age range (years) & $66-87$ & $62-88$ & $61-85$ & $61-90$ \\
Mean age $(S D)$ & $76.85(6.67)$ & $77.90(7.26)$ & $75.60(5.65)$ & $74.15(7.94)$
\end{tabular}

et al., 2009; Côté et al., 2013; Yeh et al., 2013; Christiaens et al., 2015). To determine if SC topological characteristics changed with different cortical parcellation schemes as AD progressed, SC network topological assessments were performed on a mixed ADNI cohort of $20 \mathrm{NC}, 20$ early MCI (EMCI), 20 late MCI (LMCI), and $20 \mathrm{AD}$ subjects. Finally, to explore the influence that different cortical parcellation schemes exert on the graph-based analysis of brain SC networks in AD propagation, Kruskal-Wallis tests were employed to identify group differences in network strength, global efficiency, characterized path length, cluster coefficient, $k$-core, and modularity coefficient. Additionally, linear regression analysis was used to examine the changing trajectories of rich-club coefficients for NC, EMCI, LMCI, and AD groups.

\section{MATERIALS AND METHODS}

\section{Data}

Data used in the preparation of this article were obtained from the Alzheimer's Disease Neuroimaging Initiative (ADNI) database (adni.loni.usc.edu). The ADNI was launched in 2003 as a public-private partnership, led by Principal Investigator Michael W. Weiner, MD. The primary goal of the ADNI has been to test whether serial magnetic resonance imaging (MRI), positron emission tomography (PET), other biological markers, and clinical and neuropsychological assessment can be combined to measure the progression of mild cognitive impairment (MCI) and early AD (Jack et al., 2008; Risacher et al., 2009; Petersen et al., 2010). In this study, 80 subjects were selected from the ADNI database and arranged into NC, EMCI, LMCI, and AD groups according to their ADNI classification. Table 1 shows the demographics of the participants, including age and gender. All 80 participants underwent whole-brain MRI scanning using 3T GE Medical Systems scanners. The acquisition protocol included a T1-weighted image (acquisition matrix $=256 \times 256$ $\times 196$, voxel size $=1.05 \times 1.05 \times 1.2 \mathrm{~mm}^{3}, \mathrm{TR}=6.96 \mathrm{~ms}$, $\mathrm{TE}=2.83 \mathrm{~ms})$. Furthermore, the participants were scanned with DWI echo planar imaging (EPI) protocol. Specifically, five images with no diffusion sensitization (b0 images) and 41 images along 41 diffusion directions were acquired $\left(b=1,000 \mathrm{~s} / \mathrm{mm}^{2}\right)$ with the following parameters: acquisition matrix $=128 \times 128 \times 55$, voxel size $=2.7 \times 2.7 \times 2.7 \mathrm{~mm}^{3}, \mathrm{TR}=7,200.0 \mathrm{~ms}, \mathrm{TE}=56.0 \mathrm{~ms}$.

\section{SC Network Construction}

We evaluated the influence of cortex parcellation schemes on the topological characterization of $\mathrm{AD}$ propagation by systematically varying the number of brain parcellated regions.
The cerebral cortex of each subject was parcellated into 62, 116,180 , or 333 regions, according to the parcellation templates of OASIS-TRT-20 (Klein and Tourville, 2012), AAL (TzourioMazoyer et al., 2002), HCP-MMP (Glasser et al., 2016), and Gordon-rsfMRI (Gordon et al., 2014), respectively. These four parcellation templates were all spatially normalized into Montreal Neurological Institute (MNI) space (Fonov et al., 2011), and are visualized from different views in Figure S1. Before tracking, the parcellation labels of these templates were used to segment whole brain into clusters of cortical regions. The parcellation templates were co-registered from MNI space (1 $\mathrm{mm}^{3}$ ) to DWI space via T1-weighted images using a 12-degreeof-freedom transformation matrix, using Freesurfer 6.0.0 and DSI Studio.

The construction of subject-specific SC networks requires a number of complex steps, including cortical parcellation, fiber tractography, and connection strength estimation, as shown in Figure 1. To determine the structural connectivity between each pair of cortical regions, deterministic ODF-based tractography was used. First, the eddy current effects and motion artifacts in the DWI images were corrected using the DiffusionKit toolbox (Xie et al., 2016). DWI images were then denoised using singular value decomposition and non-local means methods, as described in $\mathrm{Wu}$ et al. (2018a). Second, a model-free general q-ball imaging (GQI) reconstruction method was employed to estimate ODFs from the HARDI images, with high sensitivity and specificity to WM characteristics and pathology (Yeh and Tseng, 2011). The whole-brain fiber tracking was performed using DSI Studio software (Yeh et al., 2013), with a fractional anisotropy (FA) threshold of 0.2 and a track-turning angular threshold of $60^{\circ}$ between each two connections. Cortical connections were established between any set of cortical regions that a fiber bundle passed through or ended in. ODF-based tracking was chosen for this application, as it can resolve multiple fiber populations, including crossing, branching, and merging fibers, and thereby produces more accurate results than DT-based tracking methods (Barnett, 2009; Zhan et al., 2015b; Wu et al., 2018b). The number of reconstructed fibers between different regions were then used to define SC network edges (Hagmann et al., 2007; Houenou et al., 2007; Li et al., 2009), while each parcellated region was regarded as a network node.

\section{Network Topological Metrics}

Graph theory provides a set of measures to concisely quantify the topological properties of brain networks and describe interrelationships between brain regions of interest (ROIs) (represented by nodes in SC networks). Graph-based analysis of brain SC topological patterns allows for the quantification of a broad range of network characteristics. The most common measures used to describe the integrity of healthy or diseased brain networks include network strength, characteristic path length, efficiency, clustering coefficient, $k$-core, richclub coefficient, and modularity (Sporns, 2010). Topological characterization was performed using the GRETNA (http:// www.nitrc.org/projects/gretna/) (Wang et al., 2015) and Brain Connectivity Toolbox (BCT) toolboxes (https://sites.google.com/ site/bctnet/) (Rubinov and Sporns, 2010). The utilized network 


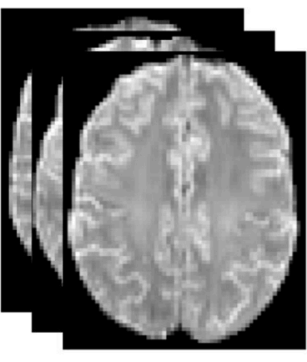

HARDI images

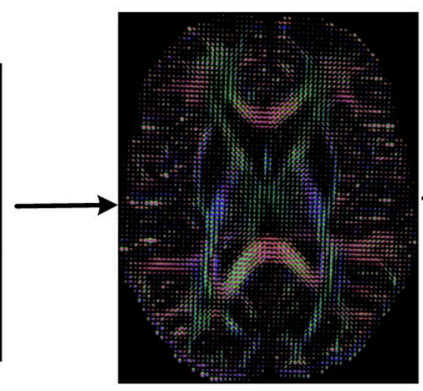

ODFs
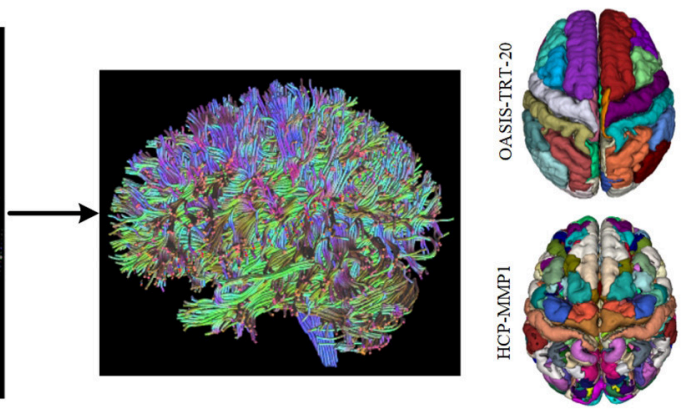

Fiber tracks

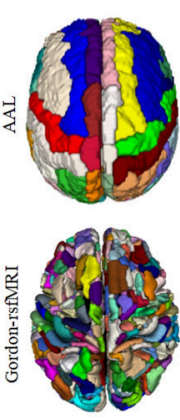

Parcellation atlases

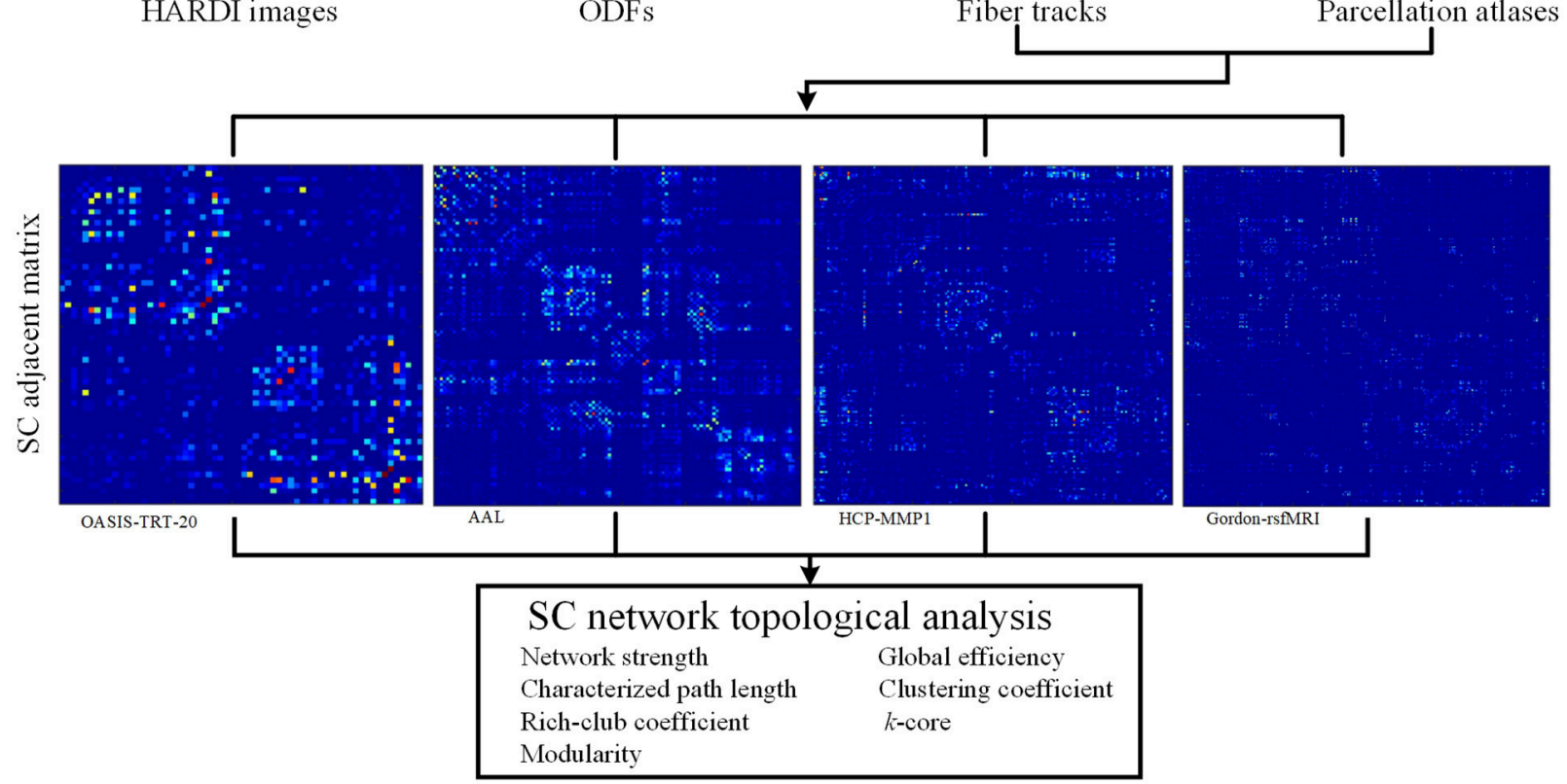

FIGURE 1 | Flowchart of SC network topological analysis. Four parcellation atlases were employed to define the nodes of the SC networks, including OASIS-TRT-20 (62 regions) (Klein and Tourville, 2012), AAL (116 regions) (Tzourio-Mazoyer et al., 2002), HCP-MMP (180 regions) (Glasser et al., 2016), and Gordon-rsfMRI (333 regions) (Gordon et al., 2014). ODFs were computed from HARDI images with a general q-ball imaging (GQI) computational model. Whole-brain tractography was performed using the DSI-Studio tool, and edges were determined by the number of neural connections between each pair of parcellated regions.

metrics are briefly described below (Cao et al., 2013; Daianu et al., 2013a, 2015).

\section{Network Strength}

For a SC network $G$ with $N$ nodes and $K$ edges, we calculated the strength of $G$ as Cao et al. (2013):

$$
S_{p}(G)=\frac{1}{N} \sum_{i \in G} S(i)
$$

where $S(i)$ is the sum of the edge weights linking to node $i$. The strength of a network is the average of the connection strengths across all of the nodes in the network. This metric reflects the extent to which network nodes are connected.

\section{Clustering Coefficient}

The clustering coefficient $C_{p}$ of a network is the average of the clustering coefficient over all nodes, which indicates the extent of local interconnectivity or cliquishness in a network (Watts and Strogatz, 1998).

$$
\mathrm{C}(i)=\frac{2}{k_{i}\left(k_{i}-1\right)} \sum_{j, k}\left(w_{i j} w_{j k} w_{k i}\right)^{1 / 3}
$$

Where $k_{i}$ is the degree of node $i$, and $w$ is connection weight. The clustering coefficient will be zero if all nodes are isolated or have just one connection (Watts and Strogatz, 1998).

$$
C_{p}=\frac{1}{N} \sum_{i \in G} C(i)
$$

\section{Characteristic Path Length}

The path length between any pair of nodes is defined as the sum of the edge lengths along this path. In this study, the length of each edge was assigned by computing the reciprocal of the 
edge weight, $\frac{1}{w_{i j}}$. The characteristic path length of $G$ was then computed as Cao et al. (2013):

$$
L_{c}(G)=\frac{1}{N(N-1)} \sum_{i \neq j \in G} L_{i j}
$$

where $L_{i j}$ defined as the shortest path between node $i$ and node $j$. This metric quantifies the ability for information to be propagated in parallel.

\section{Network Efficiency}

The global efficiency of $G$ measures the efficiency of parallel information transfer throughout the network, which can be computed as follows (Cao et al., 2013):

$$
E_{g l o b}(G)=\frac{1}{N(N-1)} \sum_{i \neq j \in G} \frac{1}{L_{i j}}
$$

where $L_{i j}$ is the shortest path length between nodes $i$ and $j$ in $G$.

\section{k-Core Decomposition}

To model the basic architecture of SC networks, a $k$-core decomposition algorithm that disentangles the hierarchical structure of the networks was proposed in Daianu et al. (2013b). This $k$-core decomposition outputs a network core that consists of highly and mutually interconnected nodes. This is accomplished by recursively removing nodes with degrees lower than $k$, such that $k$ serves as a degree threshold for nodes, ultimately identifying dense subsets of the graph.

\section{Rich-Club Coefficient}

"Rich-club" is a network property that describes how high-degree network nodes are more interconnected than would be expected by chance. The rich-club coefficient is the ratio of the number of connections among nodes of degree $k$ (or higher) to the total possible number of connections for those nodes (Daianu et al., 2015). In this study, Rich-club coefficients were calculated at a range of degree thresholds. The rich-club coefficient can be determined as:

$$
R(k)=\frac{E_{>k}}{N_{>k}\left(N_{>k}-1\right)}
$$

where $R$ is rich-club coefficient, $E_{>k}$ is the number of connections among nodes of degree $\mathrm{k}$ or higher, and $N_{>k}\left(N_{>k}-1\right)$ is the total possible number of connections if those nodes were fully connected.

\section{Modularity}

Modularity or community structure is a property that is common to brain SC networks, which divides SC network nodes into groups such that structural connections within each group are dense while connections between the groups are sparse. The study of modularity structures in SC networks can provide invaluable help in understanding and visualizing the structure of SC networks. Modularization is an optimization process in which the maximal value of $Q$ - the quantity known as modularityis obtained over all possible divisions of a network (Newman,

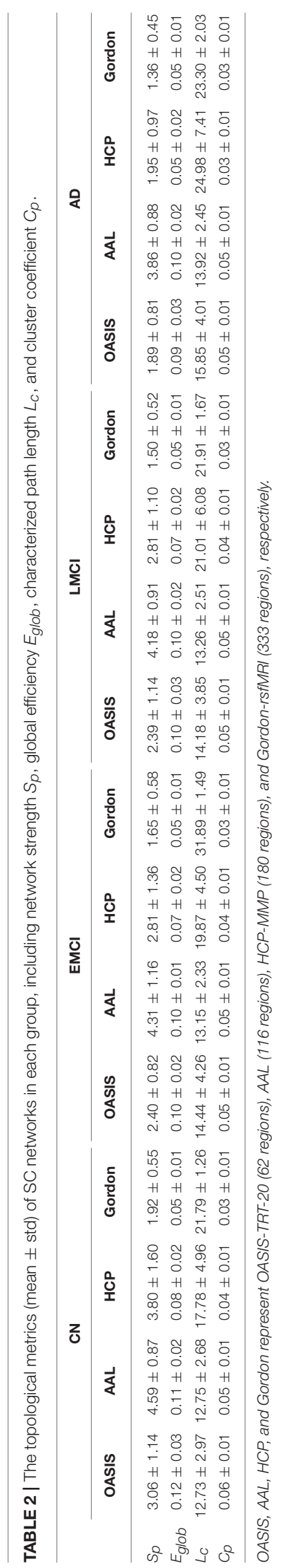


2006). Larger $Q$ values are indicative of a highly modular network organization, while lower $Q$ values indicate a more uniform network structure (Newman and Girvan, 2004). In this study, the community_louvain function of the BCT was used to calculate the modularity for the identified SC networks. The employed Louvain optimization is a simple, efficient, and easyto-implement method for identifying modules in large networks. The optimization comprises two steps. First, the method searches for small modules by optimizing modularity locally. Second, it aggregates the nodes that belong to the same module and builds a new network wherein each node represents a module identified in the first step. These steps are iterated until a maximum of modularity value is attained and a hierarchy of modules is generated (Blondel et al., 2008; Lancichinetti and Fortunato, 2009). Modularity (Q) is defined as:

$$
Q=\frac{1}{2 m} \sum_{i, j}\left[w_{i j}-\frac{k_{i} k_{j}}{2 m}\right] \delta\left(c_{i}, c_{j}\right)
$$

where $w_{i j}$ denotes the linking weight between node $i$ and node $j$; $k_{i}$, and $k_{j}$ are the sums of the weights of the edges attached to nodes $i$ and $j$, respectively; $m$ is the total link weight in the network overall; and $\delta\left(c_{i}, c_{j}\right)$ is 1 when nodes $i$ and $j$ are assigned to the same module and 0 otherwise.

\section{Topological Metric Estimation}

After SC network nodes were determined using four different parcellation schemes, ODF-based tractography was employed to calculate the structural connectivity for each subject. Connection

TABLE $3 \mid P$-values of Kruskal-Wallis testing for $S_{p}, E_{g l o b}, L_{c}$, and $C_{p}$ differences among the $\mathrm{NC}, \mathrm{EMCl}, \mathrm{LMCl}$, and AD groups.

\begin{tabular}{lllll}
\hline & OASIS & AAL & HCP & Gordon \\
\hline$S_{p}$ & $\mathbf{0 . 0 0 1 7}$ & 0.0782 & $\mathbf{0 . 0 0 0 7}$ & $\mathbf{0 . 0 0 3 2}$ \\
$E_{g / o b}$ & $\mathbf{0 . 0 0 8 0}$ & 0.8482 & $\mathbf{0 . 0 0 1 5}$ & 0.9816 \\
$L_{C}$ & 0.0637 & 0.3627 & $\mathbf{0 . 0 0 1 9}$ & $\mathbf{0 . 0 0 0 3}$ \\
$C_{p}$ & 0.0514 & 0.9988 & $\mathbf{0 . 0 2 0 7}$ & 0.9538
\end{tabular}

The significant p-values are shown in bold. OASIS, AAL, HCP, and Gordon represent OASIS-TRT-20 (62 regions), AAL (116 regions), HCP-MMP (180 regions), and GordonrsfMRI (333 regions), respectively. $S_{p}$ denotes network strength. $E_{\text {glob }}$ is global network efficiency. $L_{c}$ is characterized path length. $C_{p}$ is clustering coefficient.

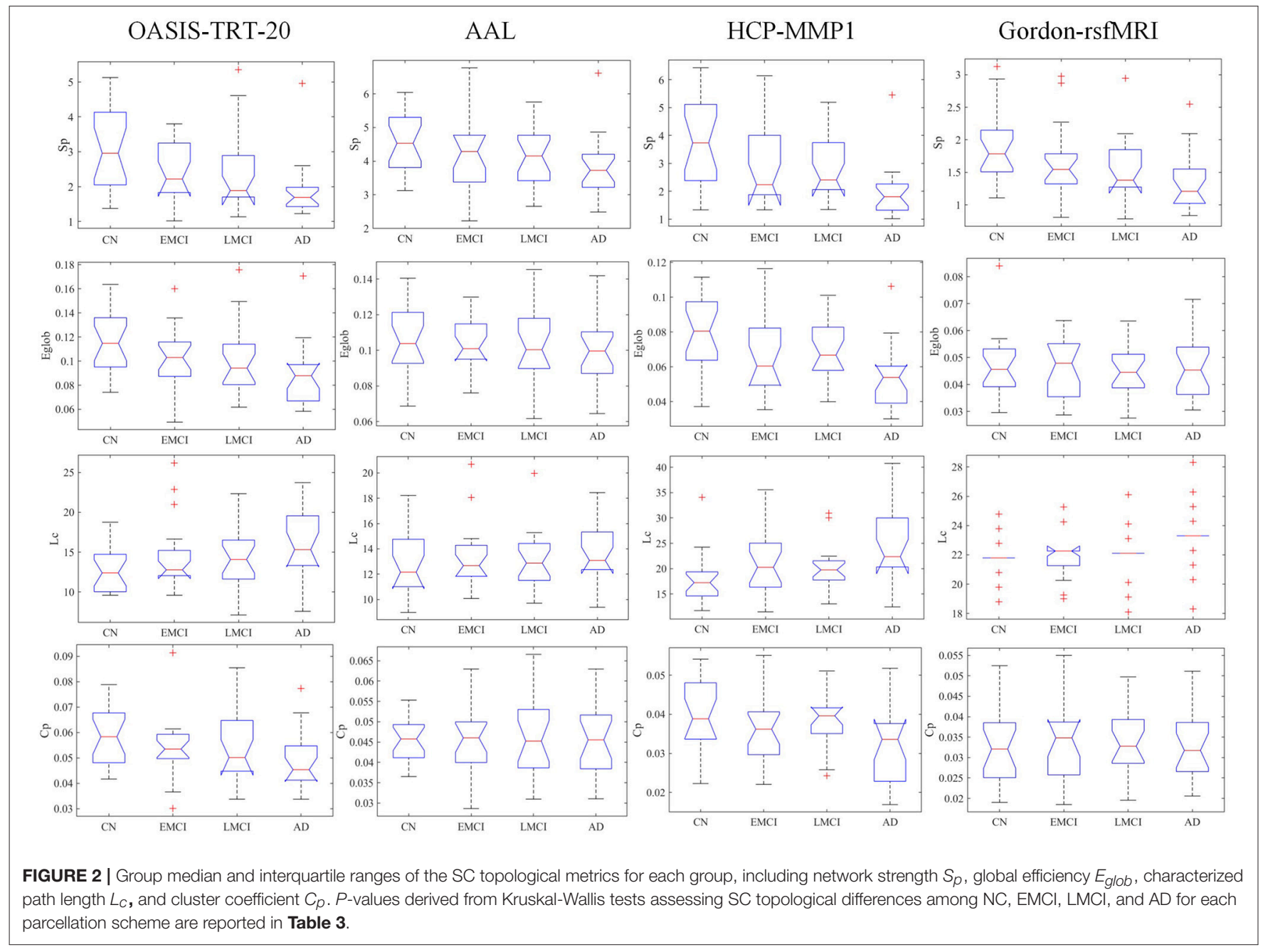


strength values were normalized from $[0,1]$ and self-connections were excluded. Group average SC matrices were computed for each parcellation scheme. Afterwards, topological measures were estimated using the codes provided in the BCT and GRETNA, including network strength, global efficiency, characterized path length, cluster coefficient, $k$-core, rich-club coefficient, and modularity. Separate from other measures, $k$-core measurement directly reflects how the SC network breaks down as cognitive impairment increases, quantifying how $\mathrm{AD}$ affects the human connectome (Daianu et al., 2013b). $k$ acts as a degree threshold for network nodes by which $k$-core decomposition creates a subnetwork that consists of highly and mutually interconnected nodes by recursively removing the nodes with degrees lower than $k$. In this study, we used $k$-core analysis to access ADrelated anatomical network changes under different whole-brain parcellation schemes including OASIS-TRT-20, AAL, HCPMMP, and Gordon-rsfMRI. When using a $k$ threshold $<17, \mathrm{AD}$ subjects cannot be discriminated from NC and MCI subjects (Daianu et al., 2015). Thus, thresholds of $k=20$ and $k=30$ are typically chosen for comparative computations. The richclub coefficient is the ratio of the number of connections among nodes of degree $k$ or higher to the total possible number of connections if those nodes were fully connected (Daianu et al., 2015). This coefficient was computed at a range of $k$ value thresholds from 17 to 39 . When the threshold is $<17$, the coefficient is close to 1 (Daianu et al., 2015). Modularity optimization is a complete subdivision of the network into nonoverlapping modules (Fortunato, 2010), which maximizes the number of within-module edges and minimizes the number of between-module edges. In this study, we used a Louvain community detection algorithm provided in BCT to achieve sub-module decomposition.

\section{Statistical Analysis}

To evaluate discriminating power for $\mathrm{AD}$ progressing phases of the network metrices corresponding to different parcellation schemes, statistical analyses were separately performed on each of them using Kruskal-Wallis tests. Additionally, a linear regression model was fitted to rich-club coefficient over a range of $k$-levels from 17 to 39 (Daianu et al., 2015), which was calculated using different whole-brain parcellation schemes. The intercepts and slopes of these regression models generally reflect the associations between rich-club coefficient and progressive AD phases. $P$ values lower than 0.05 were considered statistically significant.

\section{RESULTS}

The group-averaged SC matrices of NC, EMCI, LMCI, and $\mathrm{AD}$ groups are depicted in Figure S2. The calculated network metrics (mean \pm std) for each parcellation method are listed in Table 2, including network strength $S_{p}$, global efficiency $E_{g l o b}$, characterized path length $L_{c}$, and cluster coefficient $C_{p}$. The mean and standard deviation of group network metrics computed for each parcellation scheme are reported in Table 2, with median values and interquartile differences represented in Figure 2. $P$-values derived from Kruskal-Wallis tests assessing the SC differences among NC, EMCI, LMCI, and AD groups for each parcellation scheme are reported in Table 3 (significant values are shown in bold). Significant group differences in $S_{p}, E_{g l o b}, L_{c}$, and $C_{p}$ values were observed when HCP-MMP

TABLE $4 \mid P$-values of Kruskal-Wallis testing for $k$-core differences among the NC, $\mathrm{EMCl}, \mathrm{LMCl}$, and AD groups.

\begin{tabular}{lllll}
\hline & OASIS & AAL & HCP & Gordon \\
\hline$k=20$ & $\mathbf{0 . 0 0 3 8}$ & 0.2345 & $\mathbf{0 . 0 1 0 5}$ & $\mathbf{0 . 0 0 1 4}$ \\
$k=30$ & $\mathbf{0 . 0 0 4 0}$ & 0.1696 & $\mathbf{0 . 0 0 2 4}$ & $\mathbf{0 . 0 0 2 3}$ \\
\hline
\end{tabular}

The significant $p$-values are shown in bold. OASIS, AAL, HCP, and Gordon represent OASIS-TRT-20 (62 regions), AAL (116 regions), HCP-MMP (180 regions), and GordonrsfMRI (333 regions), respectively.

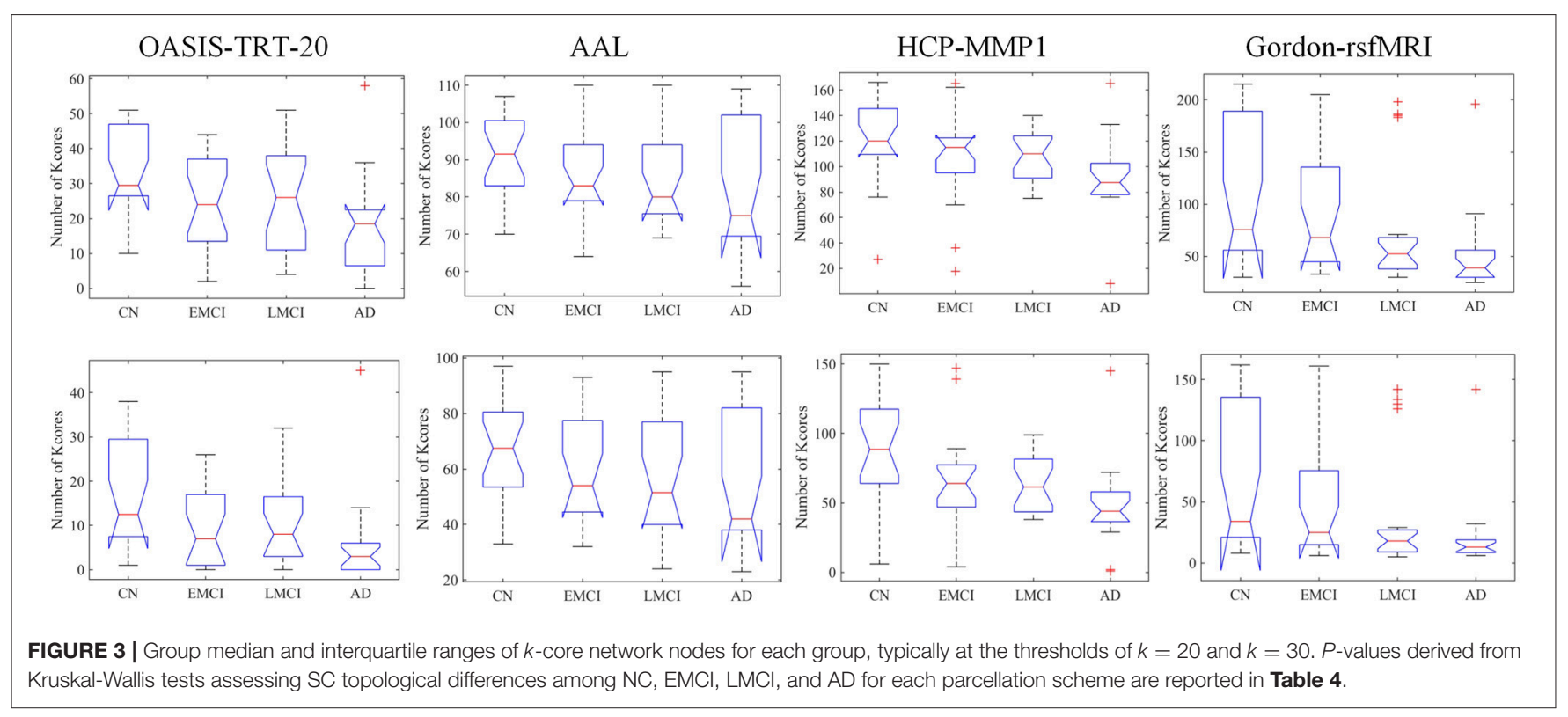




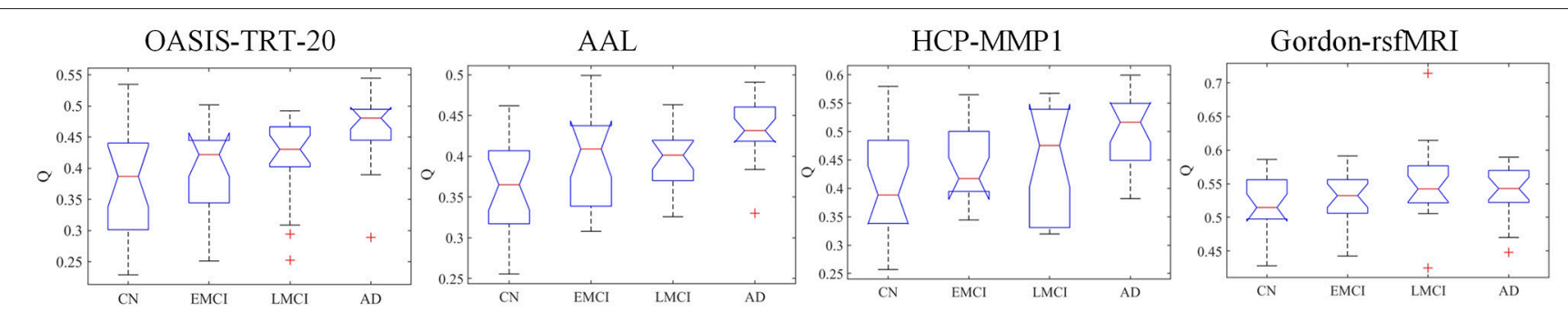

FIGURE 4 | Group median and interquartile ranges of Q coefficient of modularity structure for each group. P-values derived from Kruskal-Wallis tests assessing SC topological differences among $\mathrm{NC}, \mathrm{EMCl}, \mathrm{LMCl}$, and $\mathrm{AD}$ for each parcellation scheme are reported in Table $\mathbf{5}$.

TABLE 5 | $P$-values of Kruskal-Wallis testing for modularity differences among the $\mathrm{NC}, \mathrm{EMCl}, \mathrm{LMCl}$, and AD groups.

\begin{tabular}{lllll}
\hline & OASIS & AAL & HCP & Gordon \\
\hline Q of modularity & $\mathbf{0 . 0 0 0 4}$ & $\mathbf{0 . 0 0 0 8}$ & $\mathbf{0 . 0 1 4 8}$ & 0.1540 \\
\hline
\end{tabular}

The significant $p$-values are shown in bold. OASIS, AAL, HCP, and Gordon represent OASIS-TRT-20 (62 regions), AAL (116 regions), HCP-MMP (180 regions), and GordonrsfMRI (333 regions), respectively.

was used as parcellation scheme $(p=0.0007, p=0.0015$, $p=0.0019$, and $p=0.0207$, respectively). No group differences were found for any AAL-based SC indexes. Significant group differences were found in $S_{p}$ and $L_{c}(p=0.0032$ and $p=0.0003$, respectively) but not in $\mathrm{f} E_{\text {glob }}$ and $C_{p}$ for SC matrices constructed with Gordon-rsfMRI nodes. For OASIS-TRT-20 parcellation, significant differences in $S_{p}$ and $E_{g l o b}$ were found among the NC, EMCI, LMCI and $\mathrm{AD}$ ( $p=0.0017$ and $p=0.0080$, respectively). No significant group differences in $L_{c}$ and $C_{p}$ values were observed for OASIS parcellation.

At the typical thresholds of $k=20$ and $k=30$, Kruskal-Wallis tests were performed on the number of $k$-core network nodes of NC, EMCI, LMCI, and AD groups. Figure 3 shows group median and interquartile ranges, and the corresponding $p$-values are provided in Table 4. The results indicate that significant group differences in terms of $k$-core were detected when OASIS-TRT-20, HCP-MMP, and Gordon-rsfMRI were used as parcellation schemes $(p=0.0038 / 0.0040, p=0.0105 / 0.0024$, and $p=0.0014 / 0.0023$, respectively). For AAL parcellation, no significant group differences in $k$-core number were observed ( $p$ $=0.2345 / 0.1696)$.

The topological metric of Q quantifies the extent to which SC networks may be subdivided into clearly delineated groups. Figure 4 shows group median and interquartile ranges of modularity statistic Q across different parcellation schemes, and Table 5 shows the $p$-values with significant values in bold. Results indicate that significant group differences were detected when OASIS-TRT-20, AAL, and HCP-MMP parcellation schemes were used to define network nodes $(p=0.0004, p=0.0008$, and $p=0.0148$, respectively). For Gordon-rsfMRI parcellation, no significant group differences in Q were observed ( $p=0.1540)$.

To evaluate discriminatory efficacy of different parcellation schemes on rich-club coefficient, Kruskal-Wallis tests on the rich-club coefficient $R(k)$ at each of the $k$-levels from 17 to 39
TABLE $6 \mid P$-values of Kruskal-Wallis testing for rich-club coefficient differences among the $\mathrm{NC}, \mathrm{EMCl}, \mathrm{LMCl}$, and $\mathrm{AD}$ groups.

\begin{tabular}{|c|c|c|c|c|}
\hline$k$-level & OASIS & AAL & HCP & Gordon \\
\hline 17 & 0.0965 & 0.0002 & 0.0013 & $<0.0001$ \\
\hline 18 & 0.0045 & 0.0002 & 0.0007 & $<0.0001$ \\
\hline 19 & $<0.0001$ & 0.0002 & 0.0008 & $<0.0001$ \\
\hline 20 & $<0.0001$ & $<0.0001$ & 0.0006 & $<0.0001$ \\
\hline 21 & $<0.0001$ & 0.0002 & 0.0004 & $<0.0001$ \\
\hline 22 & $<0.0001$ & 0.0004 & 0.0005 & $<0.0001$ \\
\hline 23 & $<0.0001$ & 0.0015 & 0.0007 & $<0.0001$ \\
\hline 24 & 0.0002 & 0.0005 & 0.0004 & $<0.0001$ \\
\hline 25 & $<0.0001$ & 0.0005 & 0.0005 & $<0.0001$ \\
\hline 26 & 0.0004 & 0.0005 & 0.0003 & $<0.0001$ \\
\hline 27 & 0.0002 & 0.0002 & 0.0003 & $<0.0001$ \\
\hline 28 & 0.0001 & 0.0002 & 0.0002 & $<0.0001$ \\
\hline 29 & $<0.0001$ & 0.0001 & 0.0006 & $<0.0001$ \\
\hline 30 & $<0.0001$ & 0.0003 & 0.0009 & $<0.0001$ \\
\hline 31 & $<0.0001$ & 0.0005 & 0.0006 & $<0.0001$ \\
\hline 32 & $<0.0001$ & 0.0005 & 0.0006 & $<0.0001$ \\
\hline 33 & $<0.0001$ & 0.0006 & 0.0007 & $<0.0001$ \\
\hline 34 & $<0.0001$ & 0.0006 & 0.0005 & 0.0002 \\
\hline 35 & $<0.0001$ & 0.0010 & 0.0006 & 0.0003 \\
\hline 36 & $<0.0001$ & 0.0007 & 0.0009 & 0.0005 \\
\hline 37 & 0.0001 & 0.0008 & $<0.0001$ & 0.0008 \\
\hline 38 & $<0.0001$ & 0.0006 & 0.0008 & 0.0012 \\
\hline 39 & 0.0003 & 0.0007 & 0.0012 & 0.0032 \\
\hline
\end{tabular}

The significant $p$-values are shown in bold. OASIS, AAL, HCP, and Gordon represent OASIS-TRT-20 (62 regions), AAL (116 regions), HCP-MMP (180 regions), and GordonrsfMRI (333 regions), respectively.

(Daianu et al., 2015) were performed ( $p$-values at each $k$-level are shown in Table 6), a linear regression model was fit to $R(k)$ as it was calculated over the $k$-levels from 17 to 39 . The results in Table 6 indicate that the rich-club coefficients computed based on AAL, HCP-MMP, and Gordon-rsfMRI over the $k$-levels from 17 to 39 are significantly sensitive to the group differences across $\mathrm{NC}, \mathrm{EMCI}, \mathrm{LMCI}$, and $\mathrm{AD}$ (the corresponding $p$-values are $<0.05)$. Except for the rich-club coefficients computed based on the OASIS_TRT_20 atlas at $k$-level $=17(p=0.0965)$, the coefficients at $k$-level between 18 and 39 are able to significantly differentiate the groups (the corresponding $p$-values are $<0.05$ ). 
Figure 5 shows the linear regression fitted results which reflects the changing trend of rich-club coefficient over the $k$-levels from 17 to 39 .

\section{DISCUSSION}

To this point, evaluating the effects of brain parcellation on the topological characterization of SC networks has been a challenging task, largely due to the lack of universallyaccepted parcellation templates that can be used as a reference (Arslan et al., 2017). To provide an effective comparison, this study applied different parcellation schemes and ODF-based tractography to build SC networks for NC, EMCI, LMCI, and $\mathrm{AD}$ subjects. Four whole-brain parcellation techniques were used to define the nodes of these SC networks with different number of parcels, and connections were estimated by measuring the pairwise number of neural fiber bundles. To assess the impact of parcellation scheme on the ability to identify differences among NC, EMCI, LMCI, and AD, we explored the topological organization of SC networks. Our findings provide evidence that parcellation schemes have significant impact on topological characterization of brain structural connectivity networks in $\mathrm{AD}$ propagation.

After the topological measures were derived from subjectspecific adjacency matrices, Kruskal-Wallis tests were employed to investigate their sensitivity to the NC, EMCI, LMCI, and AD groups under each parcellation scheme. Tested measures included network strength, global efficiency, clustering coefficient, characteristic path length, $k$-core, rich-club coefficient, and modularity. We found that these measures were generally sensitive to the selection of parcellation scheme. When interpreting the SC-related results of AD-related studies, the parcellation effect on the calculated measures is a factor that needs to be taken into consideration.

Overall, characteristic path length increased with $\mathrm{AD}$ progression in all tested parcellation schemes while network strength, global efficiency, and clustering coefficient decreased, as shown in Table 2 and Figure 2. This is consistent with the results in Lo et al. (2010), Yao et al. (2010), and Daianu et al. (2013a). When the HCP-MMP (180 nodes) parcellation was used to define network nodes, the metrics, including $S_{p}, E_{g l o b}, L_{c}$, and $C_{p}$, displayed significant differences between the NC, EMCI, LMCI, and $\mathrm{AD}$ groups. In contrary, $\mathrm{AAL}$ atlas cannot discriminate group differences in terms of $S_{p}, E_{g l o b}, C_{p}, L_{c}$. The OASIS-TRT20 scheme was unable to differentiate group differences in terms of $C_{p}$ and $L_{c}$, while Gordon_rsfMRI scheme cannot recognize group differences in terms of $E_{g l o b}$ and $C_{p}$. From the results, we could conclude that network strength $S_{p}$ was most robust and sensitive to the characterization of topological deterioration in MCI and $\mathrm{AD}$, while clustering coefficient $C_{p}$ lacked robustness to whole-brain parcellation atlases. These findings align with a previous study which investigated structural connectivity and the sensitivity of network measures to the parcel number of the parcellation scheme (Zalesky et al., 2010).

$k$-core patterns in the SC networks were then explored, from which the most highly interconnected subnetworks were determined. Kruskal-Wallis test was then performed to determine if $k$-core regions remained intact or were altered by $\mathrm{AD}$ progression by eliminating the least reliable anatomical connections (Daianu et al., 2013b). In this study, we analyzed the $k$-core feature at $k=20$ and $k=30$, as $k=16$ has been reported as the minimum value at which the majority of nodes in networks would remain connected. As Daianu et al. (2013b) explored, some $k$-core nodes are lost with $\mathrm{AD}$ progression (Figure 3). We used the number of $k$-core nodes as a measure to investigate $\mathrm{AD}$-related network disruption. Significant group differences in the $k$-core patterns of the NC, EMCI, LMCI, and $\mathrm{AD}$ groups were found under the OASIS-TRT-20, HCP-MMP, and Gordon_rsfMRI parcellation schemes. Regardless of $k$-level, group difference could not be detected when using AAL atlas.

Modularity was then used to measure the extent to which a network is optimally partitioned into functional subgroups (Rubinov and Sporns, 2011). Due to the breakdown of anatomical connections, the modularity structures of the SC networks exhibited apparent alterations (Figure 4). The breakdown of global informative connections involving the medial prefrontal, posterior parietal, and insular cortices were already apparent in $\mathrm{MCI}$, suggesting that progressive damage to fiber connections begins during the predementia stages of $\mathrm{AD}$ (Acosta-Cabronero et al., 2009; Sorg et al., 2009; Sperling et al., 2010; Shao et al., 2012). AD patients then show reduced associative white matter fiber density in the cingulum, the splenium of the corpus callosum, and the superior longitudinal fasciculus (Rose et al., 2000). Coherence studies have further identified disturbed interhemispheric functional connectivity in AD (Brun and Englund, 1986; Wada et al., 1998; Delbeuck et al., 2003). According to Kruskal-Wallis testing, the NC, EMCI, LMCI, and AD groups showed significantly different $\mathrm{Q}$ values under most parcellation schemes, with the lone exception of the Gordon_rsfMRI333 parcellation. Further, our results indicate that a loss of $k$-core nodes should increase modularity (Figure 4). This supports the concept that, in addition to mediating internetwork interactions, $k$-core nodes are involved in maintaining the modular structure of functional networks through decreasing network connectivity (Hwang et al., 2017). In accordance with (Daianu et al., 2015), findings here indicate that the breakdown of anatomical connections affected by MCI and $\mathrm{AD}$ could increase the modularity coefficient.

Highly connected $k$-core nodes serve as communication hubs, facilitating integrative information processing. These hubs have high nodal degrees and tend to form a rich club-a set of nodes that are densely interconnected. The rich-club coefficient is a related but separate concept from $k$-core, as it evaluates a range of $k$-core thresholds from 17 to 39 . The rich-club coefficient is defined as the ratio of the number of connections among nodes of degree $k$ or higher to the total possible number of connections if those nodes were fully connected (Daianu et al., 2015). Significant group differences in rich-club coefficient were detected when AAL, HCP-MMP, and Gordon_rsfMRI333 parcellation schemes were used to define SC network nodes. Under the OASIS scheme, no significant group differences were detected at $k$-level $=17$. Although some conditional differences were limited, these results help better understand nodal degree alterations in AD. Finally, 

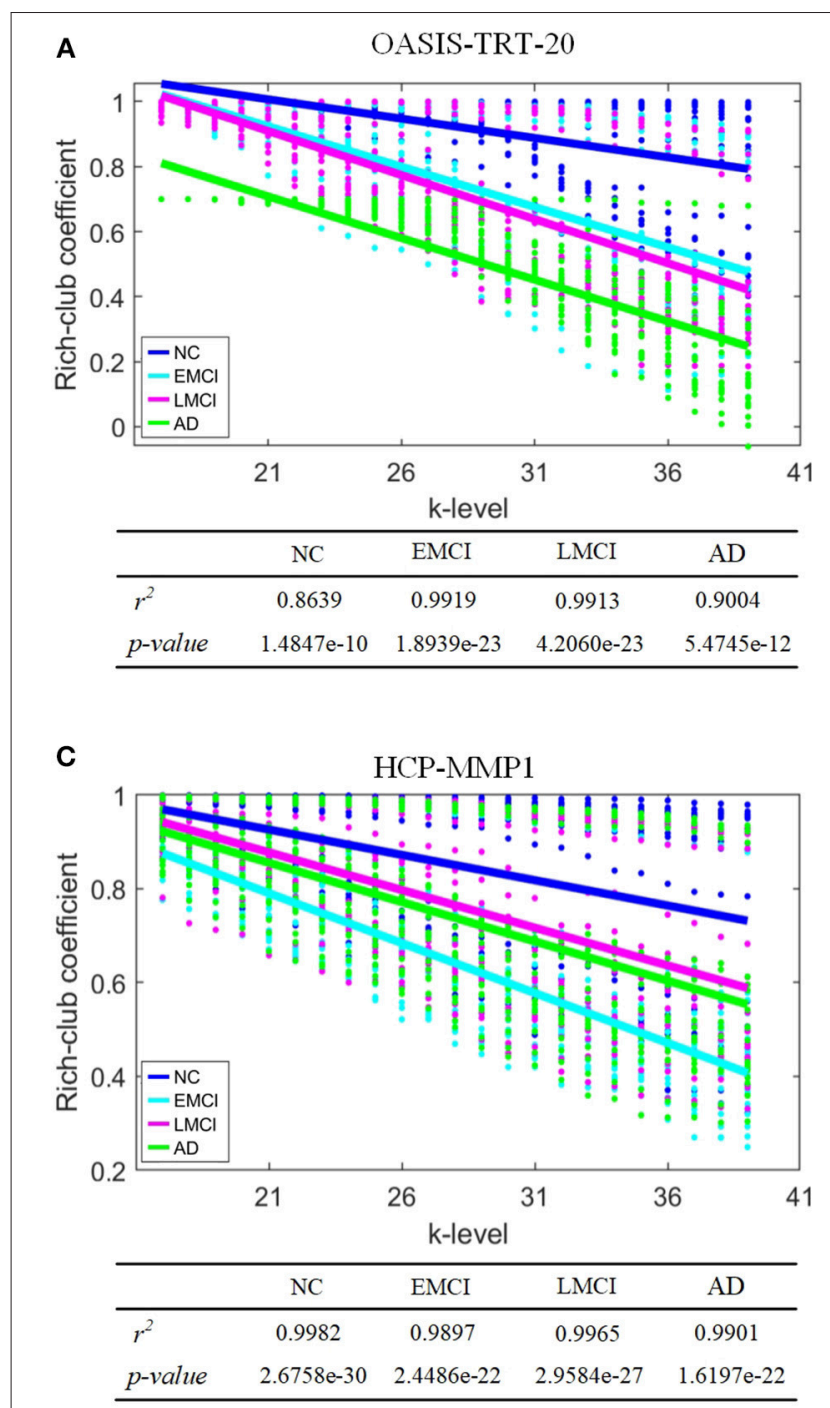

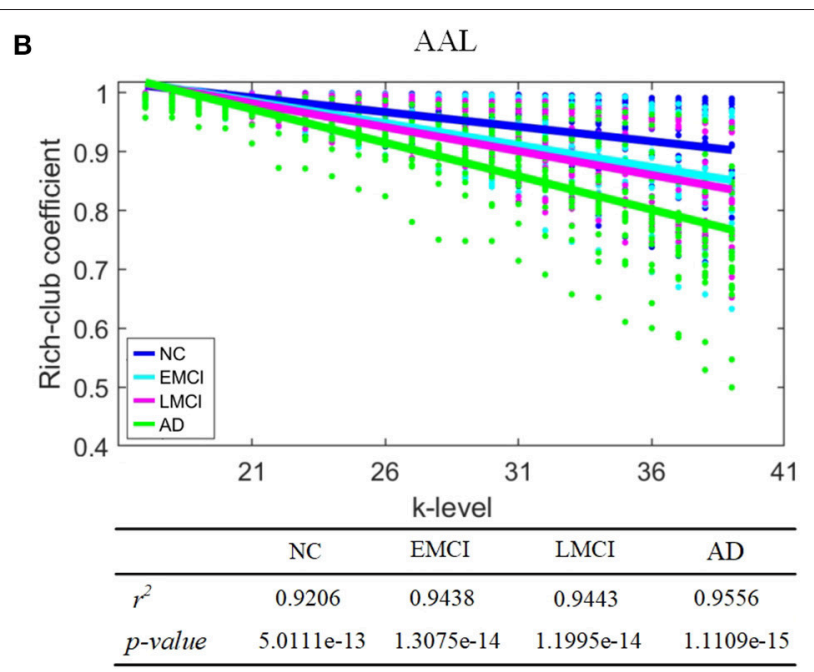

D

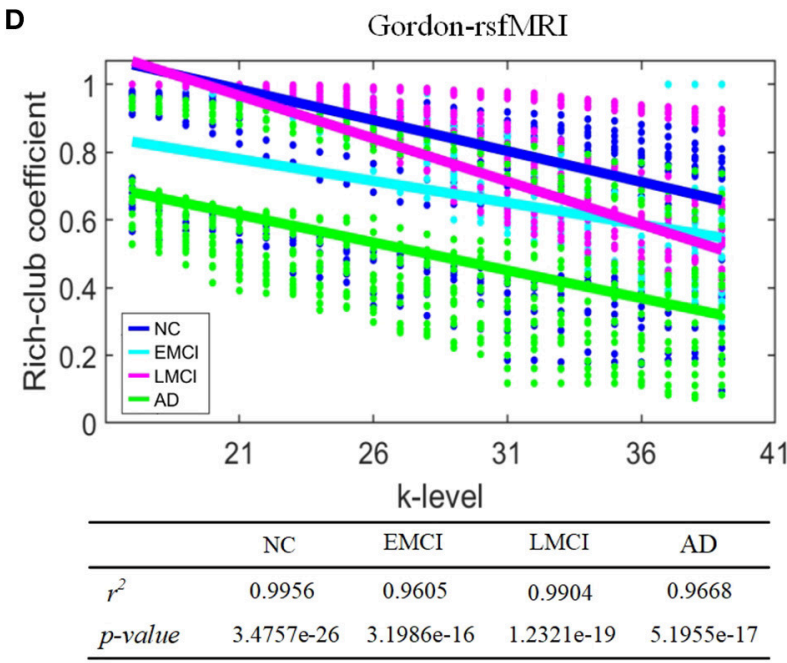

FIGURE 5 | Linear regression of rich-club coefficient over a range of $k$-level from 17 to 39. NC, EMCl, LMCl, and AD groups are represented by blue, cyan, magenta, and green colors, respectively. (A) OASIS-TRT-20, (B) AAL, (C) HCP-MMP, and (D) Gordon-rsfMRI.

the changing trends of rich-club coefficients over $k$-level was investigated using linear regression by fitting models with the metrics as predictors for $\mathrm{AD}$ propagation. The results indicate that the trends in this metric were different depending on the parcellation scheme used during SC network construction (Figure 5). Overall, rich-club coefficient changes in EMCI, LMCI, and $\mathrm{AD}$ accompany a decrease in $k$-core nodes.

From these results, it can be concluded that whole-brain parcellations exert significant influence on the topological characterization of brain structural connectivity networks in $\mathrm{AD}$ propagation. Future AD-related structural network studies should attempt to use metrics that are largely robust to the underlying parcellation scheme when attempting to predict $\mathrm{AD}$ progression. While it was not possible in this study due to limited available information, the incorporation of clinical and neuropsychological information (such as the Clinical Dementia Rating or Mini-Mental State Examination) should also be considered during analysis. Further, the effect of applied parcellation schemes should be considered during the interpretation of results, as even the most robust measures exhibit some degree of scheme-based variability. As tractography methods could greatly influence the construction of SC networks, a more sophisticated HARDI-based tractography approach may improve the credibility of SC matrices in future.

\section{CONCLUSION}

Brain parcellation influences the construction of SC network and their topological properties. This work aims to comprehensively explore effect of brain parcellation atlases on characterization of topological deterioration in MCI and AD. There is increasing evidence that widespread network disruptions exist in MCI and $\mathrm{AD}$, and that topological characterization can provide useful biomarkers for the detection of $\mathrm{AD}$ progression. In this study 
ODF-based tractography was employed to construct SC networks from a mixed cohort of $20 \mathrm{NC}, 20 \mathrm{EMCI}, 20 \mathrm{LMCI}$, and $20 \mathrm{AD}$ from ADNI under different whole-brain parcellation schemes across multiple spatial scales. The influence of parcellation scheme on the differentiation of the NC, EMCI, LEMCI, and $\mathrm{AD}$ groups was then demonstrated. Results suggest differences in the parcellation schemes used to generate SC networks affect the ability for network measures to distinguish structural differences between the NC, EMCI, LMCI, and AD groups. While this study has underlined the importance of the brain parcellation schemes in the $\mathrm{SC}$ network analysis of $\mathrm{AD}$ progression, further research is required to fully understand the relationship between SC networks and the underlying neural substrates of EMCI, LMCI, and $\mathrm{AD}$ at the network level.

\section{ETHICS STATEMENT}

In this study, 80 subjects were selected from the Alzheimer's Disease Neuroimaging Initiative (ADNI) database (http://adni. loni.usc.edu/) and arranged into $\mathrm{NC}, \mathrm{EMCI}, \mathrm{LMCI}$, and $\mathrm{AD}$ groups. The ADNI is a comprehensive, multisite longitudinal study, led by principal investigator Michael W. Weiner, M.D., that was launched as a public-private initiative in 2003 to identify the biomarkers that predict MCI and AD progression (Jack et al., 2008; Risacher et al., 2009; Petersen et al., 2010). The primary goal of ADNI is to test whether MRI, positron emission tomography (PET), and clinical/neuropsychological assessment can be combined to measure the progression of MCI and AD. All ADNI subjects gave written informed consent at enrollment for data collection, storage, and use for research.

\section{AUTHOR CONTRIBUTIONS}

ZW and YZ: study design. ZW: data acquisition. ZW, DX, TP, and YZ: analysis and interpretation, manuscript drafting, and final approval.

\section{FUNDING}

The research is supported in part by Natural Science Foundation of Zhejiang Province (LY17E070007), National Natural Science Foundation of China (51207038), China Scholarship Council, and the University of Houston. Data collection and sharing for this project was funded by the Alzheimer's Disease Neuroimaging Initiative (ADNI) (National Institutes of Health Grant U01 AG024904) and DOD ADNI (Department of Defense award number W81XWH-12-2-0012). ADNI is funded by the National

\section{REFERENCES}

Acosta-Cabronero, J., Willianms, G. B., Pengas, G., and Nestor, P. J. (2009). Absolute diffusivities define the landscape of white matter degeneration in Alzheimer's disease. Brain 133, 529-539. doi: 10.1093/brain/awp257

Arslan, S., Ktena, S. I., Makropoulos, A., Robinson, E. C., Rueckert, D., and Parisot, S. (2017). Human brain mapping: a systematic comparison of
Institute on Aging, the National Institute of Biomedical Imaging and Bioengineering, and through generous contributions from the following: AbbVie, Alzheimer's Association; Alzheimer's Drug Discovery Foundation; Araclon Biotech; BioClinica, Inc.; Biogen; Bristol-Myers Squibb Company; CereSpir, Inc.; Cogstate; Eisai Inc.; Elan Pharmaceuticals, Inc.; Eli Lilly and Company; EuroImmun; F. Hoffmann-La Roche Ltd and its affiliated company Genentech, Inc.; Fujirebio; GE Healthcare; IXICO Ltd.; Janssen Alzheimer Immunotherapy Research \& Development, LLC.; Johnson \& Johnson Pharmaceutical Research \& Development LLC.; Lumosity; Lundbeck; Merck \& Co., Inc.; Meso Scale Diagnostics, LLC.; NeuroRx Research; Neurotrack Technologies; Novartis Pharmaceuticals Corporation; Pfizer Inc.; Piramal Imaging; Servier; Takeda Pharmaceutical Company; and Transition Therapeutics. The Canadian Institutes of Health Research is providing funds to support ADNI clinical sites in Canada. Private sector contributions are facilitated by the Foundation for the National Institutes of Health (www.fnih.org). The grantee organization is the Northern California Institute for Research and Education, and the study is coordinated by the Alzheimer's Therapeutic Research Institute at the University of Southern California. ADNI data are disseminated by the Laboratory for Neuro Imaging at the University of Southern California.

\section{SUPPLEMENTARY MATERIAL}

The Supplementary Material for this article can be found online at: https://www.frontiersin.org/articles/10.3389/fnagi. 2019.00113/full\#supplementary-material

The four parcellation templates were all spatially normalized into Montreal Neurological Institute (MNI) space (Fonov et al., 2011), and are visualized from different views (left, right, top, and bottom views) in Figure $\mathbf{S 1}$.

Group-averaged SC matrices were obtained by averaging the matrices of all subjects within each group, as shown in Figure S2.

Figure S1 | Cerebral cortex parcellation schemes with different numbers of parcels, including OASIS-TRT-20 (62 regions), AAL (116 regions), HCP-MMP (180 regions), and Gordon-rsfMRI (333 regions). The character $M$ represents the number of parcellated regions. These schemes are shown from left, right, bottom, and top brain views, respectively. Full index of the parcellated regions can be found in Tzourio-Mazoyer et al. (2002), Klein and Tourville (2012), Gordon et al. (2014), and Glasser et al. (2016).

Figure S2 | The group-averaged structural connectivity matrices of NC, EMCl, $\mathrm{LMCl}$, and $\mathrm{AD}$ subjects. The strength values were normalized from $[0,1]$, and self-connections were excluded. From top to bottom, the dimensions of the adjacency matrices are $62 \times 62,116 \times 116,180 \times 180$, and $333 \times 333$, respectively. 
Bozzali, M., Falini, A., Franceschi, M., Cercignani, M., Zuffi, M., Scotti, G., et al. (2002). White matter damage in Alzheimer's disease assessed in vivo using diffusion tensor magnetic resonance imaging. J. Neurol. Neurosurg. Psychiatry 72, 742-746. doi: 10.1136/jnnp.72.6.742

Brun, A., and Englund, E. (1986). A white matter disorder in dementia of the Alzheimer type: a pathoanatomical study. Ann. Neurol. 19, 253-262. doi: 10.1002/ana.410190306

Côté, M. A., Girard, G., Boré, A., Garyfallidis, E., Houde, J. C., and Descoteaux, M. (2013). Tractometer: towards validation of tractography pipelines. Med. Image Anal. 17, 844-857. doi: 10.1016/j.media.2013.03.009

Cao, Q., Shu, N., An, L., Wang, P., Sun, L., Xia, M. R., et al. (2013). Probabilistic diffusion tractography and graph theory analysis reveal abnormal white matter structural connectivity networks in drug-naive boys with attention deficit/hyperactivity disorder. J. Neurosci. 33, 10676-10687. doi: 10.1523/JNEUROSCI.4793-12.2013

Christiaens, D., Reisert, M., Dhollander, T., Sunaert, S., Suetens, P., and Maes, F. (2015). Global tractography of multi-shell diffusion-weighted imaging data using a multi-tissue model. Neuroimage 123, 89-101. doi: 10.1016/j.neuroimage.2015.08.008

Daianu, M., Dennis, E. L., Jahanshad, N., Nir, T. M., Toga, A. W., Jack, C. R. Jr., et al. (2013a). "Alzheimer's disease disrupts rich club organization in brain connectivity networks," in 2013 IEEE 10th International Symposium on Biomedical Imaging (ISBI) (San Francisco, CA), 266-269. doi: 10.1109/ISBI.2013.6556463

Daianu, M., Jahanshad, N., Nir, T. M., Jack C. R. Jr, Weiner, M. W., Bernstein, M. A., et al. (2015). Rich club analysis in the Alzheimer's disease connectome reveals a relatively undisturbed structural core network. Hum. Brain Mapp. 36, 3087-3103. doi: 10.1002/hbm.22830

Daianu, M., Jahanshad, N., Nir, T. M., Toga, A. W., Jack C. R. Jr, Weiner, M. W., et al. (2013b). Breakdown of brain connectivity between normal aging and Alzheimer's disease: a structural k-core network analysis. Brain Connect. 3, 407-422. doi: 10.1089/brain.2012.0137

Delbeuck, X., Van der Linden, M., and Collette, F. (2003). Alzheimer' disease as a disconnection syndrome? Neuropsychol. Rev. 13, 79-92. doi: 10.1023/A:1023832305702

delEtoile, J., and Adeli, H. (2017). Graph theory and brain connectivity in Alzheimer's disease. Neuroscientist 23, 616-626. doi: $10.1177 / 1073858417702621$

Descoteaux, M., Deriche, R., Knosche, T. R., and Anwander, A. (2009). Deterministic and probabilistic tractography based on complex fibre orientation distributions. IEEE Trans. Med. Imaging 28, 269-286. doi: 10.1109/TMI.2008.2004424

Fonov, V., Evans, A. C., Botteron, K., Almli, C. R., McKinstry, R. C., and Collins, D. L. (2011). Unbiased average age-appropriate atlases for pediatric studies. Neuroimage 54, 313-327. doi: 10.1016/j.neuroimage.2010.07.033

Fortunato, S. (2010). Community detection in graphs. Phys. Rep. 486, 75-174. doi: 10.1016/j.physrep.2009.11.002

Glasser, M. F., Coalson, T. S., Robinson, E. C., Hacker, C. D., Harwell, J., and Yacoub, E. (2016). A multi-modal parcellation of human cerebral cortex. Nature 536, 171-178. doi: 10.1038/nature18933

Gordon, E. M., Laumann, T. O., Adeyemo, B., Huckins, J. F., Kelley, W. M., and Petersen, S. E. (2014). Generation and evaluation of a cortical area parcellation from resting-state correlations. Cereb. Cortex 26, 288-303. doi: 10.1093/cercor/bhu239

Hagmann, P., Kurant, M., Gigandet, X., Thiran, P., Wedeen, V. J., Meuli, R., et al. (2007). Mapping human whole-brain structural networks with diffusion MRI. PLoS ONE 2:e597. doi: 10.1371/journal.pone.0000597

Houenou, J., Wessa, M., Douaud, G., Leboyer, M., Chanraud, S., Perrin, M., et al. (2007). Increased white matter connectivity in euthymic bipolar patients: diffusion tensor tractography between the subgenual cingulate and the amygdalo-hippocampal complex. Mol. Psychiatry 12, 1001-10. doi: 10.1038/sj.mp.4002010

Hwang, K., Bertolero, M., Liu, W., and D'Esposito, M. (2017). The human thalamus is an integrative hub for functional brain networks. J. Neurosci. 37, 5594-5607. doi: 10.1101/056630

Iturria-Medina, Y., Sotero, R. C., Canales-Rodríguez, E. J., Alemán-Gómez, Y., and Melie-García, L. (2008). Studying the human brain anatomical network via diffusion-weighted MRI and graph theory. Neuroimage 40, 1064-1076. doi: 10.1016/j.neuroimage.2007.10.060
Jack, C. R., Bernstein, M. A., Fox, N. C., Thompson, P., Alexander, G., and Harvey, D. (2008). The Alzheimer's disease neuroimaging initiative (ADNI): MRI methods. J. Magn. Reson. Imaging 27, 685-691. doi: 10.1002/jmri. 21049

Jessen, F., Wolfsgruber, S., Wiese, B., Bickel, H., Mosch, E., Kaduszkiewic, H., et al. (2014). AD dementia risk in late MCI, in early MCI, and in subjective memory impairment. Alzheimer's Dementia 10, 76-83. doi: 10.1016/j.jalz.2012. 09.017

Kim, W. H., Adluru, N., Chung, M. K., Okonkwo, O. C., Johnson, S. C., Bendlin, B. B., et al. (2015). Multi-resolution statistical analysis of brain connectivity graphs in preclinical Alzheimer's disease. Neuroimage 118, 103-117. doi: 10.1016/j.neuroimage.2015.05.050

Klein, A., and Tourville, J. (2012). 101 labeled brain images and a consistent human cortical labeling protocol. Front. Neurosci. 6:171. doi: 10.3389/fnins.2012.00171

Lancichinetti, A., and Fortunato, S. (2009). Community detection algorithms: a comparative analysis. Phys. Rev. E 80:056117. doi: 10.1103/PhysRevE.80.056117

Li, Y., Liu, Y., Li, J., Qin, W., Li, K., Yu, C., et al. (2009). Brain anatomical network and intelligence. PLoS Comput. Biol. 5:e1000395. doi: 10.1371/journal.pcbi.1000395

Lo, C. Y., Wang, P. N., Chou, K. H., Wang, J., He, Y., and Lin, C. P. (2010). Diffusion tensor tractography reveals abnormal topological organization in structural cortical networks in Alzheimer's disease. J. Neurosci. 30, 16876-16885. doi: 10.1523/JNEUROSCI.4136-10.2010

Mckenna, F., Koo, B. B., and Killiany, R. (2016). Comparison of ApoE-related brain connectivity differences in early MCI and normal aging populations: an fMRI study. Brain Imaging Behav. 10, 970-983. doi: 10.1007/s11682-015-9451-z

Mueller, S. G., Weiner, M. W., Thal, L. J., Petersen, R. C., Jack, C. R., Jagust, W., et al. (2005). Ways toward an early diagnosis in Alzheimer's disease: the Alzheimer's Disease Neuroimaging Initiative (ADNI). Alzheimer's Dement. 1, 55-66. doi: 10.1016/j.jalz.2005.06.003

Newman, M. E. (2006). Modularity and community structure in networks. Proc. Natl. Acad. Sci. U.S.A. 103, 8577-8582. doi: 10.1073/pnas.0601602103

Newman, M. E., and Girvan, M. (2004). Finding and evaluating community structure in networks. Phys. Rev. E 69:026113. doi: 10.1103/PhysRevE.69.026113

Nguyen, T., Potter, T., Grossman, R., and Zhang, Y. (2018). Characterization of dynamic changes of current source localization based on spatiotemporal fMRI constrained EEG source imaging. J. Neural Eng. 15:036017. doi: 10.1088/1741-2552/aa9fb2

Nir, T. M., Villalon-Reina, J. E., Prasad, G., Jahanshad, N., Joshi, S. H., and Toga, A. W. (2015). Diffusion weighted imaging-based maximum density path analysis and classification of Alzheimer's disease. Neurobiol. Aging 36, S132-S140. doi: 10.1016/j.neurobiolaging.2014.05.037

Petersen, R. C., Aisen, P. S., Beckett, L. A., Donohue, M. C., Gamst, A. C., and Harvey, D. J. (2010). Alzheimer's disease Neuroimaging Initiative (ADNI) clinical characterization. Neurology 74, 201-209. doi: 10.1212/WNL.0b013e3181cb3e25

Proix, T., Spiegler, A., Schirner, M., Rothmeier, S., Ritter, P., and Jirsa, V. K. (2016). How do parcellation size and shortrange connectivity affect dynamics in large-scale brain network models? NeuroImage 142, 135-149. doi: 10.1016/j.neuroimage.2016. 06.016

Risacher, S. L., Saykin, A. J., Wes, J. D., Shen, L., Firpi, H. A., and McDonald, B. C. (2009). Baseline MRI predictors of conversion from MCI to probable $\mathrm{AD}$ in the ADNI cohort. Curr. Alzheimer Res. 6, 347-361. doi: 10.2174/156720509788929273

Rose, S. E., Chen, F., Chalk, J. B., Zelaya, F. O., Strugnell, W. E., and Benson, M. (2000). Loss of connectivity in Alzheimer's disease: an evaluation of white matter tract integrity with colour coded MR diffusion tensor imaging. J. Neurol. Neurosurg. Psychiatry 69, 528-530. doi: 10.1136/jnnp.69.4.528

Rubinov, M., and Sporns, O. (2010). Complex network measures of brain connectivity: uses and interpretations. Neuroimage 52, 1059-1069. doi: 10.1016/j.neuroimage.2009.10.003

Rubinov, M., and Sporns, O. (2011). Weight-conserving characterization of complex functional brain networks. Neuroimage 56, 2068-2079. doi: 10.1016/j.neuroimage.2011.03.069

Shao, J., Myers, N., Yang, Q., Feng, J., Plant, C., and Böhm, C. (2012). Prediction of Alzheimer's disease using individual structural connectivity networks. Neurobiol. Aging 33, 2756-2765. doi: 10.1016/j.neurobiolaging.2012.01.017 
Sorg, C., Riedl, V., Perneczky, R., Kurz, A., and Wohlschlager, A. M. (2009). Impact of Alzheimer's disease on the functional connectivity of spontaneous brain activity. Curr. Alzheimer Res. 6, 541-553. doi: 10.2174/156720509790147106

Sperling, R. A., Dickerson, B. C., Pihlajamaki, M., Vannini, P., LaViolette, P. S., and Vitolo, O. V. (2010). Functional alterations in memory networks in early Alzheimer's disease. Neuromol. Med. 12, 27-43. doi: 10.1007/s12017-009-8109-7

Sporns, O. (2010). Networks of the Brain. Cambridge, MA: MIT press. doi: 10.7551/mitpress/8476.001.0001

Sporns, O., Chialvo, D. R., Kaiser, M., and Hilgetag, C. C. (2004). Organization, development and function of complex brain networks. Trends Cogn. Sci. 8, 418-425. doi: 10.1016/j.tics.2004.07.008

Tzourio-Mazoyer, N., Landeau, B., Papathanassiou, D., Crivello, F., Etard, O., and Delcroix, N. (2002). Automated anatomical labeling of activations in SPM using a macroscopic anatomical parcellation of the MNI MRI single-subject brain. Neuroimage 15, 273-289. doi: 10.1006/nimg.2001.0978

Wada, Y., Nanbu, Y., Koshino, Y., Yamaguchi, N., and Hashimoto, T. (1998). Reduced interhemispheric EEG coherence in Alzheimer disease: analysis during rest and photic stimulation. Alzheimer Dis. Assoc. Disord. 12, 175-81. doi: 10.1097/00002093-199809000-00009

Wang, J., Wang, X., Xia, M., Liao, X., Evans, A., and He, Y. (2015). GRETNA: a graph theoretical network analysis toolbox for imaging connectomics. Front. Hum. Neurosci. 9:386. doi: 10.3389/fnhum.2015.00386

Watts, D. J., and Strogatz, S. H. (1998). Collective dynamics of 'small-world' networks. Nature 393, 440-442. doi: 10.1038/30918

Winblad, B., Palmer, K., Kivipelto, M., Jelic, V., Fratiglioni, L., and Wahlund, L. O. (2004). Mild cognitive impairment-beyond controversies, towards a consensus: report of the International Working Group on Mild Cognitive Impairment. J. Internal Med. 256, 240-246. doi: 10.1111/j.1365-2796.2004.01380.x

$\mathrm{Wu}$, Z., Potter, T., Wu, D., and Zhang, Y. (2018a). Denoising high angular resolution diffusion imaging data by combining singular value decomposition and non-local means filter. J. Neurosci. Methods 312, 105-113. doi: 10.1016/j.jneumeth.2018.11.020

Wu, Z., Yu, X., Liu, Y., and Hong, M. (2018b). A PSO-Powell Hybrid Method to Extract Fiber Orientations from ODF. Comput. Math. Methods Med. 2018:7680164. doi: 10.1155/2018/7680164
Xie, S., Chen, L., Zuo, N., and Jiang, T. (2016). DiffusionKit: a light one-stop solution for diffusion MRI data analysis. J. Neurosci. Methods 273, 107-119. doi: 10.1016/j.jneumeth.2016.08.011

Yao, Z., Zhang, Y., Lin, L., Zhou, Y., Xu, C., and Jiang, T. (2010). Abnormal cortical networks in mild cognitive impairment and Alzheimer's Disease. PLoS Comput. Biol. 6:e1001006. doi: 10.1371/journal.pcbi.1001006

Yeh, F. C., and Tseng, W. Y. I. (2011). NTU-90: a high angular resolution brain atlas constructed by q-space diffeomorphic reconstruction. Neuroimage 58, 91-99. doi: 10.1016/j.neuroimage.2011.06.021

Yeh, F. C., Verstynen, T. D., Wang, Y., Fernández-Miranda, J. C., and Tseng, W. Y. I. (2013). Deterministic diffusion fiber tracking improved by quantitative anisotropy. PLoS ONE 8:e80713. doi: 10.1371/journal.pone.0080713

Zalesky, A., Fornito, A., Harding, I. H., Cocchi, L., Yücel, M., Pantelis, C., et al. (2010). Whole-brain anatomical networks: does the choice of nodes matter? Neuroimage 50, 970-983. doi: 10.1016/j.neuroimage.2009. 12.027

Zhan, L., Liu, Y., Wang, Y., Zhou, J., Jahanshad, N., Ye, J., et al. (2015a). Boosting brain connectome classification accuracy in Alzheimer's disease using higher-order singular value decomposition. Front. Neurosci. 9:257. doi: 10.3389/fnins.2015.00257

Zhan, L., Zhou, J., Wang, Y., Jin, Y., Jahanshad, N., and Prasad, G. (2015b). Comparison of nine tractography algorithms for detecting abnormal structural brain networks in Alzheimer's disease. Front. Aging Neurosci. 7;48. doi: 10.3389/fnagi.2015.00048

Conflict of Interest Statement: The authors declare that the research was conducted in the absence of any commercial or financial relationships that could be construed as a potential conflict of interest.

Copyright (C) 2019 Wu, Xu, Potter, Zhang and the Alzheimer's Disease Neuroimaging Initiative. This is an open-access article distributed under the terms of the Creative Commons Attribution License (CC BY). The use, distribution or reproduction in other forums is permitted, provided the original author(s) and the copyright owner(s) are credited and that the original publication in this journal is cited, in accordance with accepted academic practice. No use, distribution or reproduction is permitted which does not comply with these terms. 